\title{
REINHOLD VON SENGBUSCH zum 70. Geburtstag am 16.2.1968
}

In der Regel bereiten die Glückwünsche zum 70. Geburtstag eines erfolgreichen Wissenschaftlers den Freunden einige Schwierigkeiten, denn eigentlich wurde alles schon einmal in vollendeter Form zum 60. oder 65. Geburtstag gesagt.

Dieses Mal aber gilt unser Gruß einem Siebzigjährigen, der noch immer voller Tatkraft und Phantasie an der Lösung neuer Probleme arbeitet.

Wohlselten hat ein Züchter oder Züchtungsforscher mit so vielen verschiedenen Kulturpflanzen gearbeitet wie von Sengbusch. Häufig hat er das Objekt und damit auch die Zuchtziele gewechselt. Aber seine Art zu arbeiten blieb seit den Versuchen mit den Süßlupinen die gleiche: Erkennen eines ökonomisch wichtigen Problems - Alkaloidfreiheit der Lupinen, hoher Fasergehalt des Hanfes, früheste Reifezeit der Tomaten, Konservierbarkeit der Erdbeeren und vieles andere mehr - , Ausarbeitung einer Schnellmethode zur Bestimmung der Merkmale und die Selektion der gewünschten Formen aus einem großen, manchmal wirklich riesigen Material. Immer wieder wurde der unverwüstliche Optimismus bestätigt und die gewünschten Formen in den Populationen gefunden.

So vielfältig die Objekte der Forschung, so vielfältig auch die entwickelten Schnellmethoden - chemisch, physikalisch, biologisch. Mit sicherem Blick wurde die leistungsfähigste Methode erkannt, bis zur letzten technischen Vollendung entwickelt, wobei Einfachheit der Anwendung die wichtigste Forderung blieb.

Niemals hat er das Züchtungsproblem isoliert gesehen. Stets sah er die Aufgabe im Komplex mit der Pflanzenkultur und der Verwertung. Die schnelle Vermehrung der aufgefundenen Lupinenmutanten verlangte ein eingehendes Studium des Lupinen- anbaus und der Saatguterzeugung. Die Züchtungsarbeit an den Tomaten erforderte umfassende Kenntnisse der Methoden der Tomatenkultur im Freiland und unter Glas. Den Zuchtarbeiten am Champignon gingen umfangreiche Versuche zur Entwicklung eines geeigneten Kultursubstrates und stets reproduzierbarer Kulturbedingungen voraus.

Nachdem jahrzehntelang landwirtschaftliche und gärtnerische Kulturpflanzen, Selbstbefruchter und Fremdbefruchter der verschiedensten Art und auch Pilze ihn zu immer neuen Arbeiten veranlaßten, hat er vor wenigen Jahren mit der Lösung eines schon lange diskutierten Problems - der Züchtung eines Karpfens ohne Zwischenmuskelgräten - begonnen. Wieder war ein ganzer Komplex zu bearbeiten. Und schon heute stehen die Spezialisten bewundernd vor den Aquarien, in denen es fast mehr Fische als Wasser gibt. Durch den Einbau der Aquarien in einen geschlossenen Wasserzyklus mit biologischer Wasserklärung konnte der unüberwindliche Schwierigkeiten bereitende ,Raumfaktor" vollkommen ausgeschlossen werden. Optimale Temperaturen wurden ermittelt und ein spezielles Fertigfutter entwickelt. So werden Gewichtszunahmen erzielt, die weit über denen von Teichkarpfen liegen, und, das Wichtigste für den Züchter, eine erheblich frühere Geschlechtsreife erreicht. Mit Hilfe von Röntgenaufnahmen wurde eine beachtliche Streuung der Grätenanzahl festgestellt und damit alle Voraussetzungen für eine schnelle Lösung der gestellten Aufgabe geschaffen.

So dürfen wir dem erfolgreichen Züchter zur Vollendung seines 70. Lebensjahres nicht nur Gesundheit und Wohlergehen, sondern auch Glück"und Erfolg bei seiner weiteren Arbeit wünschen.

\section{R. SchIck, Groß-Lüsewitz}

